\title{
Nanoscale Characterization of Li-ion Battery Cathode Nanoparticles by Atom Probe Tomography Correlated with Transmission Electron Microscopy and Scanning Transmission X-Ray Microscopy
}
A. Devaraj ${ }^{1}$, C. Szymanski ${ }^{1}$, P. Yan ${ }^{1}$, C.M. Wang ${ }^{1}$, V. Murgesan ${ }^{2}$, J. M. Zheng ${ }^{2}$, J. Zhang ${ }^{2}$, T. Tyliszczak $^{3}$, S. Thevuthasan ${ }^{4}$
[1] Environmental and Molecular Sciences Laboratory, Pacific Northwest National Laboratory, Richland WA, 99354
[2] Energy and Environmental Directorate, Pacific Northwest National Laboratory, Richland WA 99354
[3] Advanced Light Source, Lawrence Berkeley National Laboratory, Berkeley CA, 94720
[4] Qatar Energy and Environmental Research Institute, Qatar, UAE

Li-ion batteries are currently powering innumerable consumable electronic devices and are also considered as a candidate for energy storage solution for long range electric vehicles [1]. There is an ongoing effort to engineer Li-ion battery electrodes that can perform with even higher energy storage capacity along with longer term energy storage performance. Specifically next generation cathode material development is of significant focus, because cathodes amount to significant volume of the battery and share a major portion of the overall cost of the battery [1, 2]. In order to develop cathodes that can repeatedly store charge at high capacities for many cycles, a thorough understanding of their starting structure, composition and chemical state as well as the changes of the same as a function of electrochemical cycling is needed. This necessitate a correlative microscopy approach of integrating three powerful microscopy techniques capable of probing composition, structure and chemical state at nanoscale, namely atom probe tomography (APT), transmission electron microscopy (TEM) and scanning transmission $\mathrm{x}$-ray microscopy (STXM) respectively. Nanoparticle morphology of the Li-ion battery cathode materials necessitate specific modifications for the sample preparation and analysis procedures for APT as discussed below. Using this correlative microscopy approach a variety of Li-ion battery cathodes based on $\mathrm{LiMO}_{2}(\mathrm{M}=\mathrm{Mn}, \mathrm{Ni}, \mathrm{Co})$ as well as layered and spinel oxides with multiple metallic elements were investigated both before they are cycled as well as after electrochemical cycling to obtain atomic scale insight towards mechanism of capacity degradation of the Li-ion battery cathode materials as a function of electrochemical cycling.

The details of specimen preparation, APT data analysis and APT results from $\mathrm{LiMnO}_{2}$ cathode nanoparticles are discussed here. For conducting APT analysis of the cathode materials, the nanoparticles of $\mathrm{LiMnO}_{2}$ dispersed on a Silicon wafer was transferred on to the Silicon microtip array using the omniprobe nanomanipulator lift-out procedure, by just using electron beam imaging and electron beam assisted Pt deposition (Figure 1(a)). Subsequently needle shaped specimens were fabricated by annular milling in a Helios Nanolab FIB system as shown in figure 1(b). The needle specimens were analyzed in a CAMECA LEAP4000XHR laser assisted APT system using $355 \mathrm{~nm}$ wavelength UV laser at $100 \mathrm{KHz}$ pulse repetition rate and $40 \mathrm{~K}$ specimen temperature. The mass-to-charge spectra consisted of both elemental peaks of Li, $\mathrm{Mn}$ and $\mathrm{O}$ ions along with many molecular ions (figure 1c). The APT reconstruction revealed nanoscale distribution of $\mathrm{Li}, \mathrm{Mn}$ and $\mathrm{O}$ (figure 1d). These APT results were correlated with aberration corrected STEM imaging and diffraction of the same cathode nanoparticles aiding in understanding atomic scale structure. STXM imaging and NEXAFS for metal L edges ( $\mathrm{Ni}, \mathrm{Mn}$, \& Co L edges) and O K edge were performed at Advanced Light Source beamlines 5.3.2.2 and 11.0.2 to understand the chemical state of elements. The NEXAFS spectra obtained on the 
cathode materials were compared with NEXAFS spectra from standard oxide specimens to understand the exact oxidation state of individual elements. By correlating the APT reconstructions with STEM images, STXM images and NEXAFS spectra a comprehensive understanding of structure, composition and chemical state of these Li-ion battery cathodes are obtained.

\section{References}

[1] Goodenough, J. B.; Kim, Y., Challenges for Rechargeable Li Batteries. Chem Mater 2010, 22 (3), 587-603.

[2] Ellis, B. L.; Lee, K. T.; Nazar, L. F., Positive Electrode Materials for Li-Ion and Li-Batteries. Chem Mater 2010, 22 (3), 691-714.

[3] A portion of the research was performed using EMSL, a national scientific user facility sponsored by the DOE-BER and located at PNNL. The characterization work was funded by laboratory directed research and development funding as a part of chemical imaging initiative in PNNL.

[4] The Advanced Light Source is supported by the Director, Office of Science, Office of Basic Energy Sciences, of the U.S. Department of Energy under Contract No. DE-AC02-05CH11231.

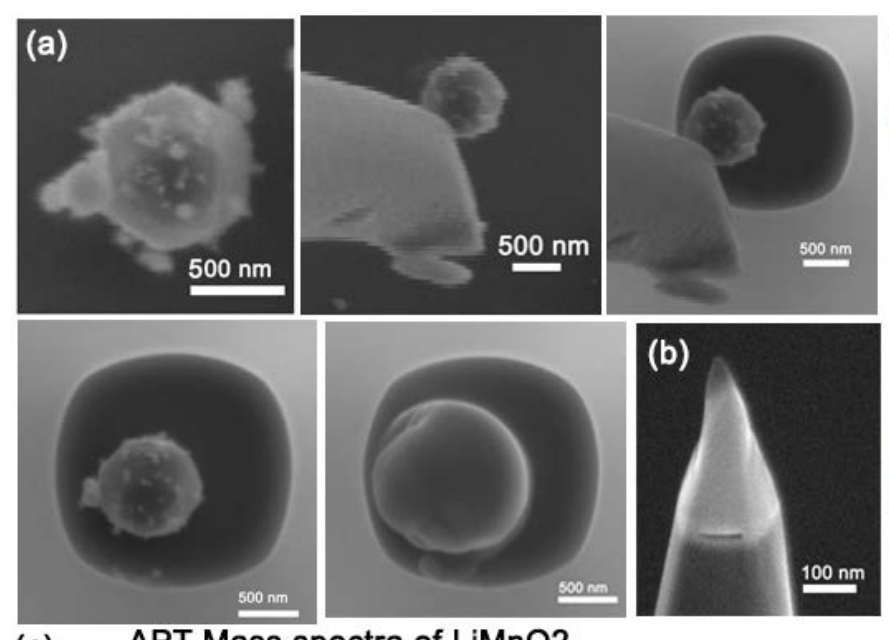

(d) APT reconstruction of $\mathrm{LiMnO2}$

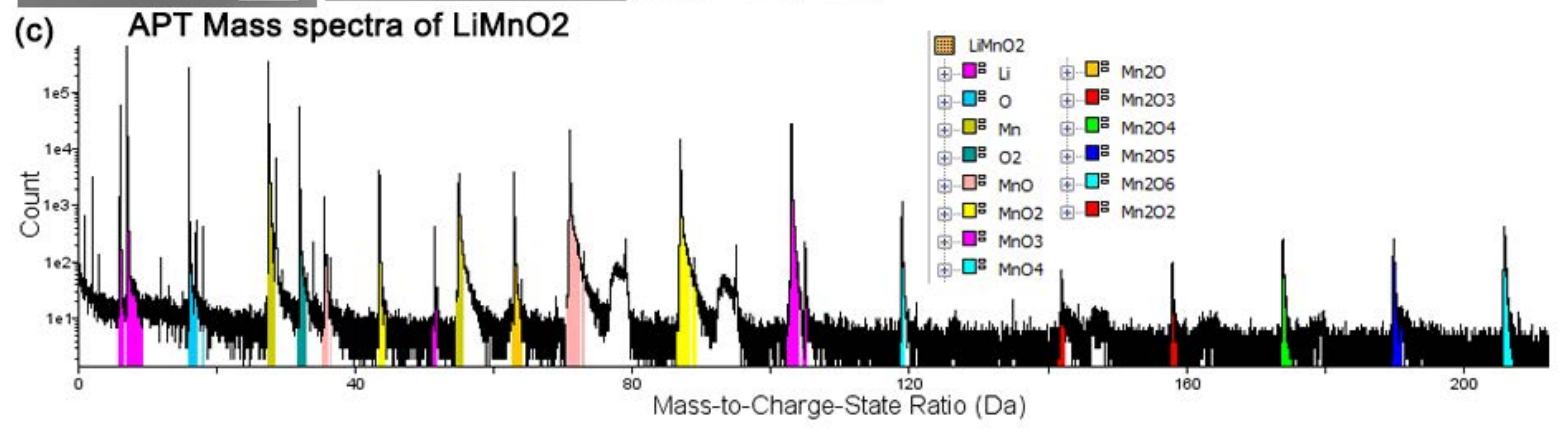

Figure 1: (a) Series of scanning electron microscopy images showing nanomanipulation of $\mathrm{LiMnO}_{2}$ nanoparticles on to a Si microtip post followed by Pt capping using electron beam assisted Pt deposition (b) final needle specimen after annular milling in FIB. (c) Mass to charge spectra of $\mathrm{LiMnO}_{2}$ obtained by laser assisted atom probe tomography indicating the different elemental and molecular ions observed (d) APT Reconstructions of $\mathrm{LiMnO}_{2}$ showing distribution of $\mathrm{Li}, \mathrm{Mn}$ and $\mathrm{O}$. 